Article

\title{
Anti-Tuberculous Activity of Treponemycin Produced by a Streptomyces Strain MS-6-6 Isolated from Saudi Arabia
}

\author{
Mahmoud A. Yassien ${ }^{1,2, *}$, Hossam M. Abdallah ${ }^{1,3}$, Ali M. El-Halawany ${ }^{1,3}$ and \\ Asif A. M. Jiman-Fatani ${ }^{4}$
}

1 Department of Natural Products and Alternative Medicine, Faculty of Pharmacy, King Abdulaziz University, P.O. Box 80260, Jeddah 21589, Saudi Arabia;

E-Mails: hmafifi2013@gmail.com (H.M.A.); ahalawany2003@yahoo.com (A.M.E.-H.)

2 Department of Microbiology and Immunology, Faculty of Pharmacy, Ain-Shams University, Cairo, Egypt

3 Department of Pharmacognosy, Faculty of Pharmacy, Cairo University, Cairo 11562, Egypt

4 Department of Medical Microbiology and Parasitology, Faculty of Medicine, King Abdulaziz University, P.O. Box 80260, Jeddah 21589, Saudi Arabia.; E-Mail: afatani@kau.edu.sa

* Author to whom correspondence should be addressed; E-Mail: myassien61@yahoo.com; Tel.: +966-508-319-951; Fax: +966-026-951-696.

Academic Editor: Derek J. McPhee

Received: 21 December 2014 / Accepted: 27 January 2015 / Published: 2 February 2015

\begin{abstract}
A Streptomyces strain MS-6-6 with promising anti-tuberculous activity was isolated from soil samples in Saudi Arabia. The nucleotide sequence of its 16S rRNA gene (1426 bp) evidenced a 100\% similarity to Streptomyces mutabilis. Through an anti-tuberculous activity-guided approach, a polyketide macrolide was isolated and identified as treponemycin (TP). The structure of the isolated compound was determined by comprehensive analyses of its 1D and 2D NMR as well as HRESI-MS. In addition to the promising anti-tuberculous activity $(\mathrm{MIC}=13.3 \mu \mathrm{g} / \mathrm{mL}$ ), TP showed broad spectrum of activity against the Gram positive, Gram negative strains, and Candida albicans. Improvement of TP productivity $(150 \%)$ was achieved through modification in liquid starch nitrate medium by replacing $\mathrm{KNO}_{3}$ with corn steep liquor and yeast extract or tryptone, and removing $\mathrm{CaCO}_{3}$ and $\mathrm{K}_{2} \mathrm{HPO}_{4}$. The follow up of TP percentage as well as its metabolites profile for each media was assessed by LC/DAD/MS.
\end{abstract}

Keywords: Streptomyces mutabilis; treponemycin; anti-tuberculous activity 


\section{Introduction}

Tuberculosis (TB) is one of the most serious infectious diseases. One-third of the world's population is infected with Mycobacterium tuberculosis. One of the most significant challenges in treating TB patients is the development of microbial resistance to many of the currently used anti-TB drugs [1].

According to the WHO estimates, the incidence of all forms of TB and its related complications was increased by $6.2 \%$ between 1990 and 2004. In Saudi Arabia, the highest level of TB infection was reported in Jeddah (31.1\%) followed by Riyadh (23.2\%) [2]. Accordingly, there is great interest in obtaining novel antibiotics with promising anti-tuberculous activity.

The genus Streptomyces, filamentous soil bacteria, have been described as the greatest source of the commercially available antibiotics [3-5]. Streptomyces are also reported to produce other valuable bioactive secondary metabolites acting as antitumor agents, immunosuppressive agents and enzymes [6-8]. Different types of anti-tuberculous antibiotics are produced by Streptomyces sp. The first one is streptomycin, discovered in 1943. Other anti-tuberculous antibiotics produced by Streptomyces include D-cycloserine and kendomycin that produced from S. garyphalus and S. violaceoruber, respectively $[9,10]$. Moreover, the microbial production of secondary metabolites including antibiotics is affected greatly by nutritional condition [11]. The medium supplies nutrients for growth, energy, building of the cell substances and biosynthesis of the desired fermentation products. The sources of carbon and nitrogen in the medium are of particular importance, since microbial cells and fermentation products are composed largely of these two elements [12]. Therefore, the composition of the fermentation medium should be taken in consideration during establishment of the suitable fermentation experiments to maximize the production of secondary metabolites.

This work describes the genetic identification of Streptomyces strain(s) with promising anti-tuberculosis activity collected from Saudi Arabia soil, purification and structure determination of the bioactive agent, evaluation of the antimicrobial activity of the purified compound, and improvement of bioactive compound productivity.

\section{Results and Discussion}

\subsection{Screening of Anti-Tuberculous Activity of Streptomyces Isolates}

A total of 492 Streptomyces isolates were obtained from 93 soil samples collected from different localities in the Western Region of Saudi Arabia. The anti-tuberculous activity of the isolates was evaluated against $M$. tuberculosis (ATCC 25177) by cup-diffusion techniques. The results showed that 155 of the tested isolates have different degrees of anti-tuberculous activity (Supplementary Table S1) from which eight isolates have promising anti-tuberculous activity (Table 1). These isolates were assessed for their cytotoxicty against normal human fetal lung fibroblast cell line (MRC-5). The obtained results (Table 1) showed that all the tested Streptomyces extracts have weak cytotoxic effects $\left(\mathrm{IC}_{50} \geq 240 \mu \mathrm{g} / \mathrm{mL}\right)$. Accordingly, the isolate coded MS-6-6 with the strongest anti-tuberculous activity was selected for further studies. 
Table 1. The anti-tuberculous activity of the selected Streptomyces extracts and their cytotoxic activities against normal human fetal lung fibroblast cell line (MRC-5).

\begin{tabular}{cccc}
\hline Strain Number & $\begin{array}{c}\text { Inhibition Zone } \\
(\mathbf{m m})\end{array}$ & $\begin{array}{c}\text { Equivalent Activity to Rifampicin } \\
(\mathbf{m g} / \mathbf{L})\end{array}$ & $\begin{array}{c}\text { Cytotoxic Activity }\left(\mathbf{I C}_{\mathbf{5 0}}\right) \\
(\boldsymbol{\mu g} / \mathbf{m L})\end{array}$ \\
\hline MS-6-6 & 29 & 0.08 & 280 \\
MS-18-4 & 20 & 0.03 & 315 \\
MKS-9-3 & 20 & 0.03 & 275 \\
JS-8-4 & 20 & 0.03 & 315 \\
JS-9-4 & 24 & 0.05 & 265 \\
JS-22-9 & 27 & 0.06 & 240 \\
GS-42-1 & 24 & 0.05 & 320 \\
ES-15-3 & 22 & 0.04 & 260 \\
\hline
\end{tabular}

\subsection{Identification of Streptomyces Strain MS-6-6}

According to the cultural, physiological, and microscopical properties of the selected isolate MS-6-6, it was identified as a member of the genus Streptomyces (Supplementary Figures S1 and S2, Tables S2-S4). The nucleotide sequence of the 16S rRNA gene (1426 bp), obtained from the MS-6-6 isolate was aligned with all presently available $16 \mathrm{~S}$ rRNA gene sequences in the GeneBank databases. The results showed high similarity (98\%-100\%) to the Streptomyces $16 \mathrm{~S}$ rRNA genes (Accession no. NR 044139.1). Phylogenetic analysis using the 16S DNA gene sequence suggests the strain is similar to Streptomyces mutabilis (Figure 1).

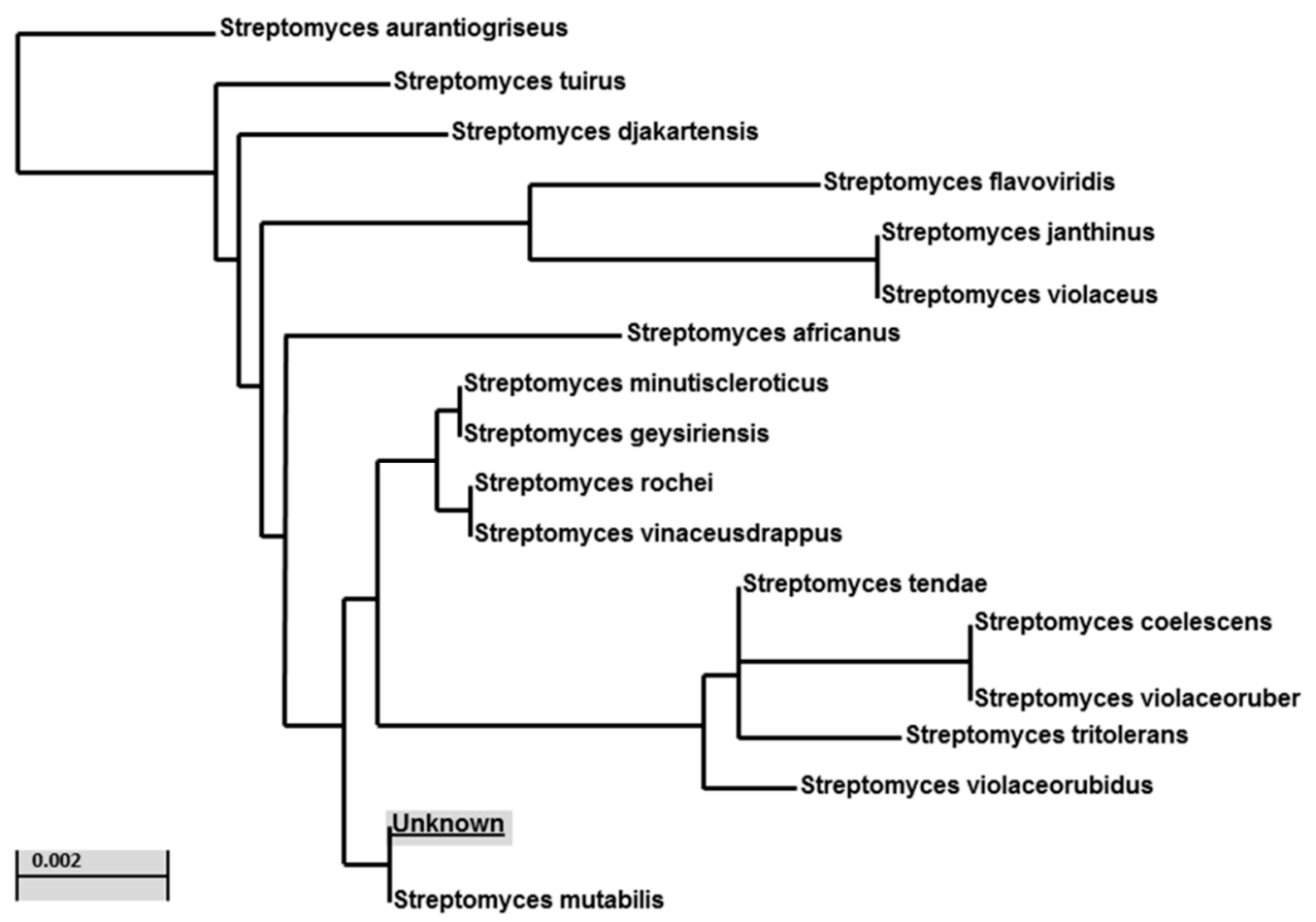

Figure 1. Phylogenic relationships between Streptomyces strain MS-6-6 and related species based on partial nucleotide sequence (1426 bp) of the 16S rRNA gene. The tree was constructed using the neighbor-joining method. The scale bar indicates a 0.002 substitution per nucleotide position. 


\subsection{Isolation and Structure Elucidation of the Bioactive Compound from the Streptomyces Mutabilis}

The antimicrobial activity of the fraction solutions was evaluated and the results revealed that fraction A-3 showed promising antimicrobial activity against the tested Gram-positive, Gram-negative bacteria, C. albicans and M. tuberculosis (zones of inhibition ranged between 15 and $24 \mathrm{~mm}$ ), while A-2 fraction exhibited a weak antimicrobial activity (range of inhibition zones 11-15 mm). However, no antimicrobial activity was obtained by testing fraction A-1 (Supplementary Table S5).

Chromatographic purification of fraction A-3 resulted in the isolation of one compound MYA-3 in pure form as a white amorphous powder $\left(4.8 \mathrm{mg}\right.$ ). The molecular formula was deduced to be $\mathrm{C}_{28} \mathrm{H}_{43} \mathrm{NO}_{6}$ from HRESI-MS which displayed a molecular ion peak at $\mathrm{m} / z 488.2874$ for $[\mathrm{M}-\mathrm{H}]^{-}$in negative ion mode and a molecular ion peak at $m / z 512.3406$ for $[\mathrm{M}+\mathrm{Na}]^{+}$in positive mode. ${ }^{1} \mathrm{H}-\mathrm{NMR}$ spectra of MY-A3 displayed four doublet methyl protons $\left(\delta_{\mathrm{H}} 0.80,0.84,0.85,1.03, J=6 \mathrm{~Hz}\right)$ for methyls attached to $\mathrm{C}_{5}$, $\mathrm{C}_{4}, \mathrm{C}_{8}$, and $\mathrm{C}_{10}$ respectively, three deshielded protons $\left(\delta_{\mathrm{H}} 3.86 m, 4.10 \mathrm{~d}, J=9.6 \mathrm{~Hz}\right.$, and 5.0, $m$ ) for $\mathrm{H}_{3}$, $\mathrm{H}_{11}$ and $\mathrm{H}_{17}$ respectively (Table 2). Moreover, three olefinic protons $\left(\delta_{\mathrm{H}} 6.20 \mathrm{~m}, 6.40 \mathrm{~m}\right.$, and $\left.6.80 \mathrm{~d}, J=10\right)$ corresponding to $\mathrm{H}_{15}, \mathrm{H}_{14}$ and $\mathrm{H}_{13}$, respectively, were observed. Furthermore, ${ }^{13} \mathrm{C}-\mathrm{NMR}$ and DEPT spectra revealed the presence of 28 carbons; four methyls, eight methylenes, 12 methane and four quaternary carbons. The spectrum displayed two characteristic peaks at 116.1 and 176.1 for $\alpha, \beta$-unsaturated nitrile and carboxylic carbons, respectively. Complete correlations between carbons and protons were achieved through two-dimensional NMR spectra, including, COSY, HSQC, HMBC and NOESY (Supplementary Figures S3-S7). All spectroscopic data proved that compound MYA-3 belongs to the polyketide macrolide group and identified it as treponemycin (TP) (borrelidin) [13-20]. Although, TP was first isolated from Streptomyces rochei, it was subsequently identified in other Streptomyces species such as $S$. californicus, $S$. rimosus, and $S$. heilongjiangensis [16-19], but this is the first time production of TP by $S$. mutabilis has been reported.

Table 2. ${ }^{1} \mathrm{H}$ - and ${ }^{13} \mathrm{C}-\mathrm{NMR}, \mathrm{COSY}$ and HMBC spectral data of compound (MYA-3) $\left(\mathrm{CDCl}_{3}\right)$.

\begin{tabular}{ccccc}
\hline Position & $\mathbf{H}$ & $\mathbf{C}$ & COSY & HMBC (H with C) \\
\hline 1 & - & 172.1 & - & - \\
2 & $2.42,2.32 m$ & $39.3\left(\mathrm{CH}_{2}\right)$ & 3.86 & $172.1,69.9,35.1$ \\
3 & $3.86, m$ & $69.9(\mathrm{CH})$ & $2.32,2.42$ & $43,172.1$ \\
4 & $1.67, \mathrm{~m}$ & $35.1(\mathrm{CH})$ & $3.86,1.25,0.84$ & $69.9,39.3,27.2$ \\
5 & $0.92,1.25$ & $43.1\left(\mathrm{CH}_{2}\right)$ & $1.67,1.57$ & $18.23,47.8,69.9$ \\
6 & 1.57 & $27.2(\mathrm{CH})$ & $1.10,0.97,1.25$ & $26.3,35.1$ \\
7 & $0.97,1.10$ & 47.8 & $1.62,1.57$ & $37.5,43.1$ \\
8 & 1.62 & $26.3(\mathrm{CH})$ & $0.85,1.03,1.1$ & $65.3,27.2$ \\
9 & $1.03,0.76$ & $37.5(\mathrm{CH})$ & $1.85,1.62$ & $14.9,73.2,26.3$ \\
10 & 1.85 & $65.3(\mathrm{CH})$ & $4.1,1.03$ & $118.1,26.3$ \\
11 & $4.10, d, J=9.6$ & $73.2(\mathrm{CH})$ & 1.85 & $116.1,37.47,143.9,14.9$ \\
12 & - & 118.1 & - & - \\
13 & $6.80, d, J=10.8$ & $143.9(\mathrm{CH})$ & 6.40 & $118.1,138.5,73.2,126.9$ \\
14 & $6.40, m$ & $126.9(\mathrm{CH})$ & $6.20,6.80$ & $116.1,35.9,143.9$ \\
15 & $6.20, m$ & $138.5(\mathrm{CH})$ & $6.40,2.56$ & $143.9,35.9,76.6$ \\
\hline
\end{tabular}


Table 2. Cont.

\begin{tabular}{ccccc}
\hline Position & H & C & COSY & HMBC (H with C) \\
\hline 16 & $2.56,2.59, m$ & $35.9\left(\mathrm{CH}_{2}\right)$ & $5.0,6.20$ & $126.9,138.5,76.5,45.9$ \\
17 & $5.0, m$ & $76.5(\mathrm{CH})$ & $2.56,2.59,2.70$ & $172.1,138.5,29.6,35.9$ \\
18 & $2.70, t$ & $45.9(\mathrm{CH})$ & $1.30,1.97,2.51$ & $176.1,76.5,47.8,29.6$ \\
19 & $1.97,1.30 m$ & $29.6\left(\mathrm{CH}_{2}\right)$ & $2.70,5.0,2.51$ & $76.5,47.8,31.1,25.2$ \\
20 & $1.83,1.79$ & $25.2\left(\mathrm{CH}_{2}\right)$ & $1.30,2.03,1.90$ & $45.9,47.8$ \\
21 & $2.03,1.90 m$ & $31.1\left(\mathrm{CH}_{2}\right)$ & $1.83,1.79,2.51$ & $176.1,45.9,47.8$ \\
22 & $2.51, m$ & $47.8(\mathrm{CH})$ & $1.90,2.03,2.70$ & $176.1,76.5,45.9,29.6,31.1$ \\
$\mathrm{CH}_{3}$ at $\mathrm{C}_{4}$ & $0.84, d, J=6$ & 17.0 & - & $35.1,43.1,69.9$ \\
$\mathrm{CH}_{3}$ at $\mathrm{C}_{6}$ & $0.80, d, J=6$ & 18.2 & - & $27.2,47.8,43.1$ \\
$\mathrm{CH}_{3}$ at $\mathrm{C}_{8}$ & $0.85, d, J=6$ & 20.2 & - & $26.3,37.5,47.8$ \\
$\mathrm{CH}_{3}$ at $\mathrm{C}_{10}$ & $1.04, d, J=6$ & 14.9 & - & $65.3,73.2$ \\
$\mathrm{CN}$ & - & 116.1 & - & - \\
$\mathrm{COOH}$ & - & 176.1 & - & - \\
\hline
\end{tabular}

\subsection{Antimicrobial Activity of Treponemycin}

The results showed that TP has a strong antimicrobial activity with the MICs ranges of 1.7-16.7 and 8.3-26.7 $\mu \mathrm{g} / \mathrm{mL}$ against the tested Gram-positive, and Gram-negative strains, respectively. In addition, it has promising activity against M. tuberculosis ATCC 25177 and C. albicans with MIC values of 4.15 and $13.3 \mu \mathrm{g} / \mathrm{mL}$, respectively (Table 3). Previous biological screening indicated that TP exhibits antiviral [20] and antibacterial activities [21], which presumably arise from its inhibition of threonyl-tRNA synthetase and protein synthesis [22]. It also displays potent antimitotic properties at low concentrations due to inhibition of cyclindependent kinase. None of the previous studies observed any anti-tuberculous activity of TP.

Table 3. Antimicrobial activity of the purified antimicrobial agent, treponemycin, produced by Streptomyces isolate MS-6-6.

\begin{tabular}{cc}
\hline Organisms & MIC $(\boldsymbol{\mu g} / \mathbf{m L})$ \\
\hline Mycobacterium tuberculosis & 4.17 \\
Staphylococcus epidermidis & 1.7 \\
Streptococcus pyogenes & 2.1 \\
Bacillus subtilis & 2.9 \\
Escherichia coli & 8.3 \\
Clostridium perfringens & 16.7 \\
Brucella melitensis & 16.7 \\
Pseudomonas aeruginosa & 26.7 \\
Proteus mirabilis & 11.3 \\
Candida albicans & 13.3 \\
\hline
\end{tabular}

MIC is the mean of three repeated experiments. 


\subsection{Improvement of Treponemycin Productivity}

\subsubsection{Improvement of Antimicrobial Activity}

Due to the importance of TP and its interesting biological activities, many attempts have been made to prepare it through chemical synthesis. The enantioselective total synthesis of TP is highly complicated and expensive, consequently a simpler method is vital $[13,21,23]$. S. mutabilis, isolated in the present study, has the ability to produce promising levels of TP during the fermentation process. In addition, the present study established a suitable system for purification of TP from the fermentation supernatant. In industrial microbiology, more that $70 \%$ of the produced antibiotics depend on Streptomyces spp. [5]. Therefore, from an economic view it is more interesting to depend on the fermentation of $S$. mutabilis for microbial production of TP.

The composition of the fermentation medium is of principal importance in the design of successful laboratory experiments [11]. Therefore, improvement of the microbial production of TP was performed through nutritional modification by changing the growth media and detection of the level of TP production through measuring the antimicrobial activity of growth supernatant. Accordingly, different fermentation media (Table 4) were tested for their suitability to improve the TP productivity of S. mutabilis.

Table 4. Ingredients of different fermentation media.

\begin{tabular}{|c|c|c|c|c|c|c|c|c|}
\hline Ingredients $(\mathrm{g} / \mathrm{L})$ & M1 * & M2 & M3 & M4 & M5 & M6 & M7 & M8 \\
\hline CSL & - & - & 20.0 & 20.0 & 20.0 & 10.0 & 10.0 & 20.0 \\
\hline Yeast extract & - & 10.0 & 10.0 & - & - & 10.0 & - & - \\
\hline Peptone & - & - & - & 10.0 & - & - & - & - \\
\hline Tryptone & - & 10.0 & - & & 10.0 & - & 10.0 & - \\
\hline Soluble starch & 20.0 & 10.0 & 20.0 & 20.0 & 20.0 & - & - & 10.0 \\
\hline Glucose & - & 10.0 & - & - & - & 20.0 & 10.0 & 10.0 \\
\hline $\mathrm{KNO}_{3}$ & 2.0 & - & - & - & - & - & - & - \\
\hline $\mathrm{CaCO}_{3}$ & 3.0 & - & - & - & - & - & - & - \\
\hline $\mathrm{MgSO}_{4} 7 \mathrm{H}_{2} \mathrm{O}$ & 0.5 & 0.5 & 0.5 & 0.5 & 0.5 & 0.5 & 0.5 & 0.5 \\
\hline $\mathrm{NaCl}$ & 0.5 & 0.5 & 0.5 & 0.5 & 0.5 & 5.0 & 5.0 & 5.0 \\
\hline $\mathrm{K}_{2} \mathrm{HPO}_{4}$ & 1.0 & - & - & - & - & - & - & - \\
\hline $\mathrm{FeSO}_{4} 7 \mathrm{H}_{2} \mathrm{O}$ & 0.01 & 0.01 & 0.01 & 0.01 & 0.01 & - & - & - \\
\hline $\mathrm{MnCl}_{2}$ & 0.01 & 0.01 & 0.01 & 0.01 & 0.01 & - & - & - \\
\hline $\mathrm{ZnSO}_{4} 7 \mathrm{H}_{2} \mathrm{O}$ & 0.01 & 0.01 & 0.01 & 0.01 & 0.01 & - & - & - \\
\hline
\end{tabular}

The results (Table 5) revealed that the maximum antimicrobial activity of $S$. mutabilis was obtained by using M3 and M5 media, whereby the size of the inhibition zones was increased by approximately 1.2-1.35 fold more than that produced by using the original medium (M1, liquid starch nitrate medium). M3 and M5 are modified forms of the M1 medium made by replacing $\mathrm{KNO}_{3}$ with corn steep liquor (CSL) and yeast extract or tryptone, and removing $\mathrm{CaCO}_{3}$ and $\mathrm{K}_{2} \mathrm{HPO}_{4}$. The use of organic nitrogen sources, such as CSL and yeast extract or tryptone, is much better than inorganic nitrogen source, as $\mathrm{KNO}_{3}$, for microbial production of secondary metabolites as due to their complex nature, organic nitrogenous compounds act as a slow release nitrogen sources which play important roles in the high 
productivity of secondary metabolites such as antibiotics. The suitability of using organic compounds as nitrogen sources for antibiotic production was reported by other investigators [24].

Table 5. TP titer and antimicrobial activity of $S$. mutabilis supernatant using different fermentation media.

\begin{tabular}{cccccc}
\hline \multicolumn{5}{c}{ Inhibition Zones (mm) } & TP Titer \\
\hline Media & M. tuberculosis & S. epidermisis. & E. coli & C. albicans. & mg/L \\
M1 & 24 & 18 & 16 & 14 & 0.45 \\
M2 & 17 & 15 & 14 & 12 & 0.38 \\
M3 & 29 & 24 & 21 & 18 & 0.67 \\
M4 & 26 & 21 & 18 & 15 & 0.50 \\
M5 & 28 & 22 & 19 & 16 & 0.68 \\
M6 & 17 & 15 & 14 & 12 & 0.40 \\
M7 & 17 & 15 & 14 & 11 & 0.36 \\
M8 & 22 & 17 & 15 & 13 & 0.45 \\
\hline
\end{tabular}

\subsubsection{LC-DAD/ESI-MS Analysis of TP and Its Metabolites in Different Culture Media}

The fermentation supernatants of the modified media that showed promising antimicrobial activity were selected to determine the titers of TP on the basis of LC-DAD/MS analysis calibrated with an authentic TP standard. All the tested media caused production of TP $(\mathrm{m} / \mathrm{z} 488.1$ at $9.3 \mathrm{~min})$ with different concentrations (Table 5) in addition to other metabolites (T1-T4) (Figures 2 and 3). The highest titer of TP was produced by using M3 and M5 media, in which TP was the major metabolite with approximate concentration range $0.67-0.68 \mathrm{mg} / \mathrm{L}$. Accordingly, the use of M3 and M5 media increased the TP titer by approximately 1.5 fold as compared to that of the original medium (M1).<smiles>[R]C(=C(C#N)C=CCCC(OC(=O)CC([R])C(C)CC(C)CC(C)CC(C)C)C1([2H])CCCC1[R9])C(C)C</smiles>

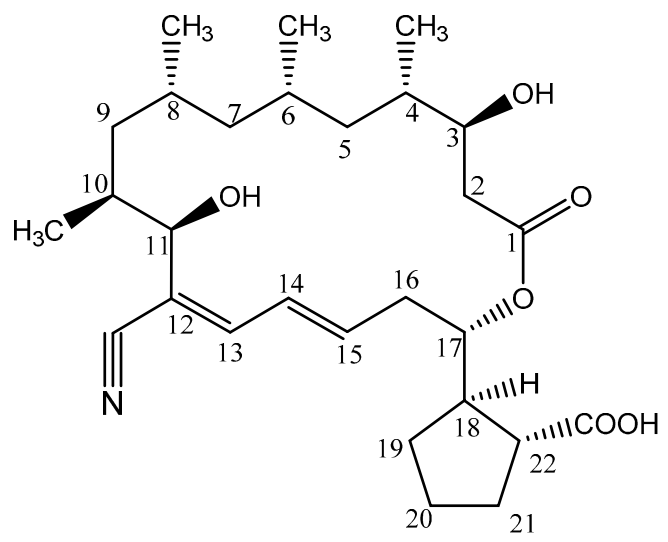

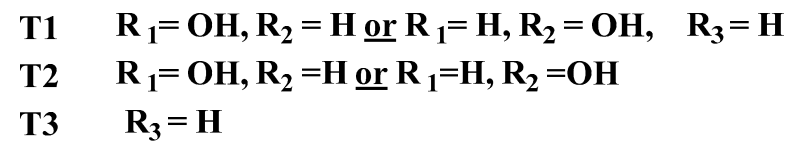

MYA3

Figure 2. Structure of trepenomycin (MYA-3) and its metabolites (T1-T3). 


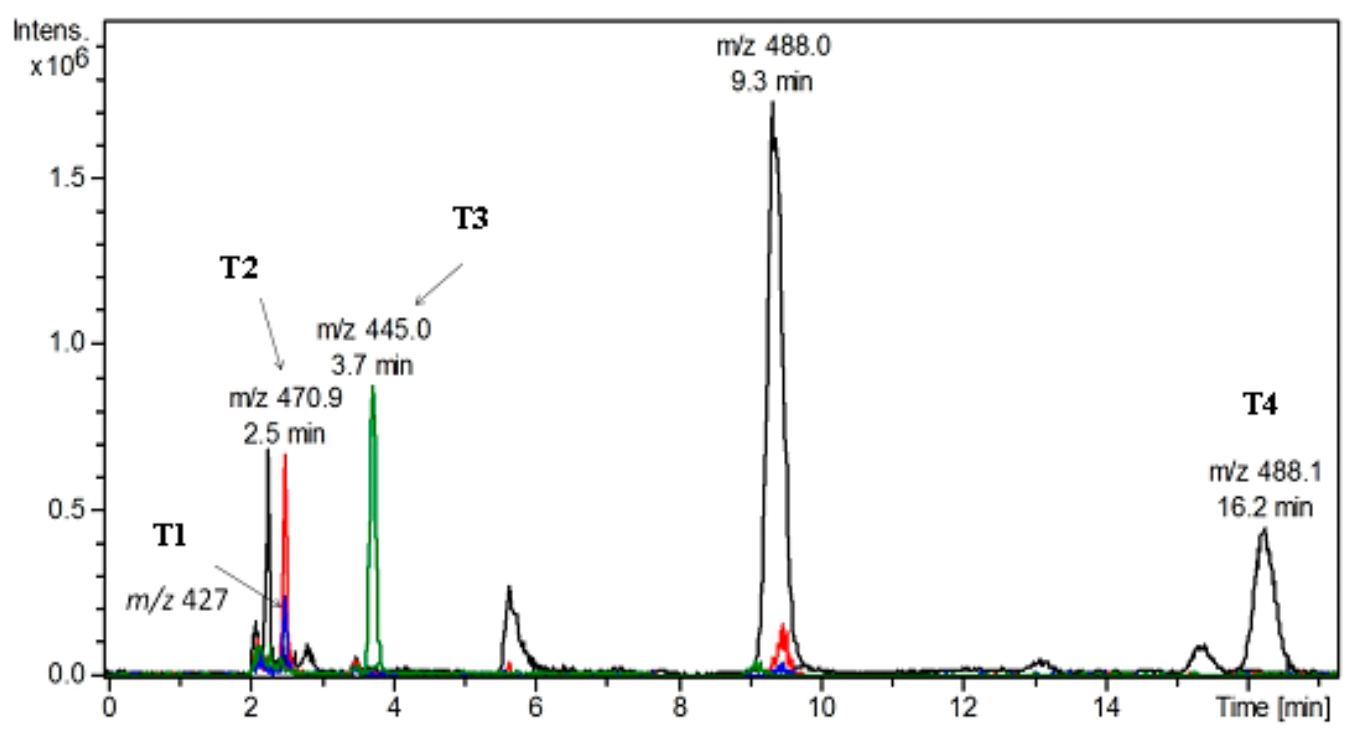

Figure 3. Representative (overlay) of extracted ion chromatograms (EIC) of treponemycin and its metabolites in different culture media.

\subsubsection{Identification of Metabolites}

Metabolite profiling of the tested media revealed the presence of TP in addition to four major related metabolites (T1-T4) at $m / z 426\left[\mathrm{M}-\mathrm{COOH}-\mathrm{H}_{2} \mathrm{O}\right]^{-}, m / z 471\left[\mathrm{M}-\mathrm{H}_{2} \mathrm{O}\right]^{-}, m / z 445\left[\mathrm{M}-\mathrm{CO}_{2}\right]^{-}$radicle ion, and $m / z 488$ [Iso-TP] $]^{-}$, respectively.

T1 showed a molecular ion peak at $m / z 426$ indicating the loss of carboxylic group ( $45 \mathrm{u}$ ) and hydroxyl moiety $(17 \mathrm{u})$ (either from position 3 or 11) (Figure 4). T2 with a molecular ion peak at $\mathrm{m} / \mathrm{z} 471$ was identified as dehydroxytrepenomycin (due to loss of a hydroxyl group from position 3 or 11). Moreover, T3 showed a molecular ion peak at 445 indicating the loss of a carboxylic acid group. Finally T4 could be tentatively identified as an isomer of TP as it shows the same molecular ion at $\mathrm{m} / z 488$ (Figure 4).

Metabolites T1 and T2 were produced in M3 medium, metabolite T3 was produced in M4 and M5 media, while metabolite T4 was produced in M2 and M6-M8 media. Moreover, media M2, M6, M7 showed variable concentrations of metabolite T2. It was reported that nitrile, lactone and probably the hydroxyl functions are essential for the antimicrobial activity of TP [25]. Therefore, the increase in antibacterial activity due to media change could be mainly due to the increase of TP concentration, as the other metabolites (T1-T4) are lacking the functional groups necessary for activity.

\section{Experimental Section}

\subsection{Microorganisms}

Mycobacterium tuberculosis (ATCC 25177), Staphylococcus epidermidis (ATCC 12228), Streptococcus pyogenes (ATCC 19615), Bacillus subtilis (ATCC 6633), Escherichia coli (ATCC 25922), Pseudomonas aeruginosa (ATCC 27853), Proteus mirabilis (ATCC 14153), Brucella melitensis 16M (ATCC 23456), Clostridium perfringens (ATCC 19404), and Candida albicans (ATCC 10231) were used. All test standard strains, except Candida albicans and Mycobacterium tuberculosis, were maintained onto Mueller Hinton Agar slants. C. albicans was maintained on Sabouraud dextrose agar 
slants, while M. tuberculosis was maintained on Lowenstein Jensen medium and Middlebrooks 7H9 agar medium. Standard strains, except M. tuberculosis, were stored in skimmed milk or $15 \%$ glycerol in Brain Heart Infusion at $-86^{\circ} \mathrm{C}$.

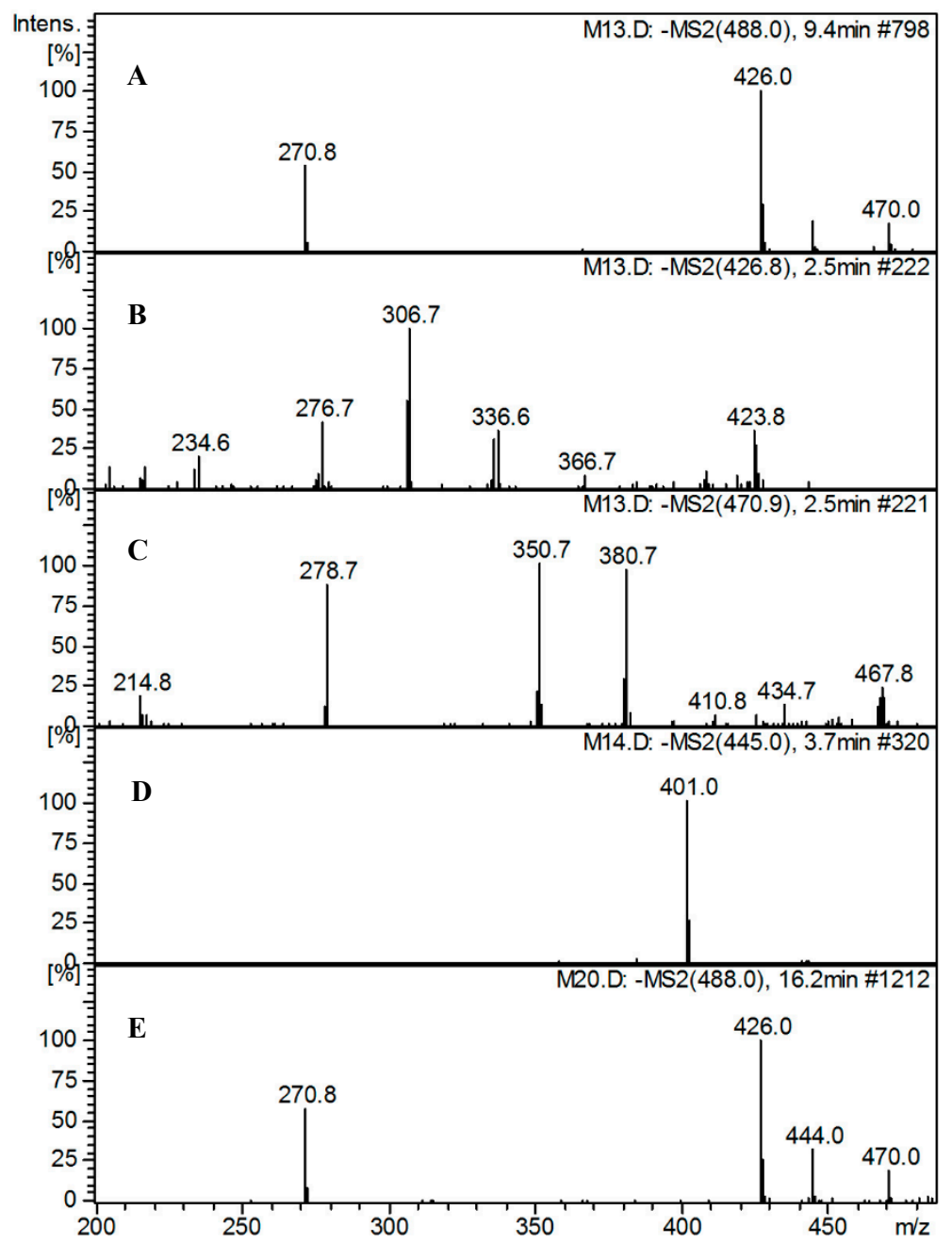

Figure 4. Mass spectra of compound MYA-3 and its detected metabolites. (A) MS2 spectra of compound MYA-3; (B) MS2 spectra of T1; (C) MS2 spectra of T2; (D) MS2 spectra of metabolite T3; (E) MS2 spectra of metabolite T4.

\subsection{Isolation of Streptomyces Strains}

Isolation of Streptomyces was carried out as described by Jaradat et al. [26]. In brief, soil suspensions (1\%) were prepared and plated onto the surface of starch-nitrate agar medium ( $20 \mathrm{~g}$ soluble starch, $2.0 \mathrm{~g}$ $\mathrm{KNO}_{3}, 1.0$ g K $2 \mathrm{HPO}_{4}, 0.5 \mathrm{~g} \mathrm{MgSO}_{4}, 0.5 \mathrm{~g} \mathrm{NaCl}, 3.0 \mathrm{~g} \mathrm{CaCO}$, $0.01 \mathrm{~g} \mathrm{FeSO}_{4}, 0.01 \mathrm{~g} \mathrm{MnCl}$, $0.01 \mathrm{~g}$ $\mathrm{ZnSo}$, $20 \mathrm{~g}$ agar per liter) and incubated at $30{ }^{\circ} \mathrm{C}$. After 5 to 7 days of incubation, colonies having the typical culture characteristics of Streptomyces (leathery or fibrous colonies appearing as granular powders with hard texture) were picked and purified by streak-plate technique on the same medium. Purified isolates were maintained onto starch-nitrate agar slants at $4{ }^{\circ} \mathrm{C}$ and subcultured every month. 


\subsection{Evaluation of Antimicrobial Activity}

\subsubsection{Fermentation and Preparation of Streptomyces Clear Supernatants}

Colonies from 4- to 5-days old starch nitrate cultures were suspended in normal saline solution and appropriately diluted to give a final count of $1-2 \times 10^{5} \mathrm{CFU} / \mathrm{mL}$ determined by viable count technique. Aliquots $(0.5 \mathrm{~mL})$ of Streptomyces spore suspensions $\left(1-5 \times 10^{5} \mathrm{CFU} / \mathrm{mL}\right)$ were used to inoculate liquid starch nitrate medium $(50 \mathrm{~mL})$ in $250 \mathrm{~mL}$ Erlenmeyer flasks and incubated in an rotary shaking-incubator (S19R-2, Sheldon Manufacturing Inc., Cornelius, OR, USA) at $200 \mathrm{rpm}$ and $30{ }^{\circ} \mathrm{C}$ for $96 \mathrm{~h}$. After incubation, a known volume of culture broth was centrifuged at $6000 \times \mathrm{g}$ for 20 min to obtain a clear supernatant and the cell pellet was collected to determine the dry cell weight.

\subsubsection{Bacterial and Fungal Susceptibility Testing}

Antimicrobial susceptibility testing was done according to the standardized cup-diffusion technique [27]. In brief, colonies from overnight cultures of the tested standard strains were suspended in saline solution and appropriately diluted to match the turbidity of standard 0.5 on McFarland scale, diluted in saline (1:100) and used for seed inoculation of $25 \mathrm{~mL}$ agar plates (Mueller-Hinton agar for bacterial strains or Sabouraud dextrose agar for $C$. albicans) to the final count of $2-5 \times 10^{5} \mathrm{CFU} / \mathrm{mL}$ as determined by viable count technique. Aliquots $(200 \mu \mathrm{L})$ of the tested samples (Streptomyces clear supernatant, the prepared fractions from the lyophilized supernatant or the bioactive compound) were evaluated by as described. Each experiment was carried out in triplicate and mean value was calculated. Ciprofloxacin (for antibacterial activity) and fluconazole (for antifungal activity) were used as positive control.

\subsubsection{Evaluation of Anti-Tuberculous Activity}

Preparation of Bacterial Suspension

Colonies from 14 to 21 days old culture of M. tuberculosis (ATCC 25177) were transferred into sterile glass vial containing $2.5 \mathrm{~mL}$ of Middlebrooks $7 \mathrm{H} 9$ fresh broth with glass beads. After vortexing for $1 \mathrm{~min}$ and waiting for $15 \mathrm{~min}$, the supernatant was transferred to another tube and diluted with the same medium to match the turbidity of McFarland 1.0 standard (equivalent to $3 \times 10^{8} \mathrm{CFU} / \mathrm{mL}$ ). The final suspension was diluted 1:20 of broth and used for inoculation of assay plates.

\section{Anti-Tuberculous Activity by Cup-Diffusion Techniques}

Aliquots $(100 \mu \mathrm{L})$ of the prepared $M$. tuberculosis suspension were used for surface inoculation of a Middlebrooks 7H10 agar medium (Difco, Detroit, MI, USA) supplemented with 10\% OADC (oleic acid-albumin-dextrose-catalase). Aliquots $(200 \mu \mathrm{L})$ of the tested samples were dripped in the prepared cups. The plates were incubated at $35^{\circ} \mathrm{C}$ under $5 \% \mathrm{CO}_{2}$ for 14 days. After incubation, the anti-tuberculosis activities were detected by observing the inhibition zones around the cups [28]. Each experiment was carried out in triplicate and mean value was calculated. 
Evaluation of Anti-Tuberculous Activity by REMA Plate Assay

The REMA plate assay was carried out as described by [29]. Briefly, $100 \mu \mathrm{L}$ of 7H9-S broth (7H9 broth $+10 \%$ OADC $+0.5 \%$ glycerol $+0.1 \%$ casitone) was dispensed in each well of a sterile flat-bottom 96-well plate, and serial twofold dilutions of the tested samples were prepared directly in the plate. One hundred microliters of inoculum, prepared as described, was added to each well. A growth control and a sterile control were also included for each isolate. Sterile water was added to all perimeter wells to avoid evaporation during the incubation. The plate was covered, sealed in a plastic bag, and incubated at $37{ }^{\circ} \mathrm{C}$ under a normal atmosphere. After 7 days of incubation, $30 \mu \mathrm{L}$ of resazurin solution $(0.02 \%)$ was added to each well, and the plate was reincubated overnight. A change in color from blue to pink indicated the growth of bacteria, and the MIC was defined as the lowest concentration of drug that prevented this change in color. Rifampicin was used as a positive control.

\subsection{Evaluation of Cytotoxic Activity}

In vitro cytotoxic activity of the tested samples was evaluated against normal human epithelial cell line MRC-5 as described by [30]. In such experiment, each treatment was carried out in triplicate. Drug free wells were used as negative control. Doxrubicine was used as a positive control.

\subsection{Isolation and Structure Elucidation of the Bioactive Compound}

\subsubsection{General}

NMR experiments were performed in $\mathrm{CDCl}_{3}$ on a DRX-600 spectrometer (Bruker BioSpin, Billerica, MA, USA). Peak positions are reported relative to TMS. Mass spectra were measured on Acquity UPLC system (Waters, Milford, MA, USA) using a MicroTOF-Q hybrid quadrupole time-of-flight mass spectrometer (Bruker Daltonics, Billerica, MA, USA). Column chromatographic separation (CC) was performed on Diaion ${ }^{\circledR}$ HP-20 (Merck, Darmstadt, Germany), silica gel 60 for column Chromatography (70-230 mesh, Merck, Darmstadt, Germany). TLC was performed on pre-coated TLC plates with silica gel 60 F 254 (Merck, Darmstadt, Germany).

\subsubsection{Biologically Guided Isolation of Active Compounds}

Freeze dried powder of MS-6-6 growth supernatant was dissolved in water, and fractionated on Diaion HP-20 using water, methanol/water and methanol to give fractions A-1, A-2, and A-3, respectively. The solvent was evaporated under vacuum to give fractions A-1 (30 g), A-2 (20 g) and A-3 (2 g). Antimicrobial activity of each fraction was evaluated $(2 \mathrm{mg} / \mathrm{mL})$ against the tested standard strains. The bioactive fraction (A-3) was chromatographed over silica gel column $(25 \times 2 \mathrm{~cm}, 50 \mathrm{~g})$, using petroleum ether/ethyl acetate $(1: 1)$ as an eluent to afford active compound MY-A3. The structure of the active compound was determined by comprehensive analyses of its 1D and 2D NMR, HRESI-MS and comparison with previously reported data. Compound MY-A3 was isolated as a white amorphous powder, HR-ESIMS $m / z 512.3406[\mathrm{M}+\mathrm{Na}]^{+}$(calc. for $\mathrm{C}_{28} \mathrm{H}_{43} \mathrm{NO}_{6} \mathrm{Na}$ ) in positive mode and $\mathrm{m} / \mathrm{z} 488.2874$ $[\mathrm{M}-\mathrm{H}]^{-}$(calc. for $\mathrm{C}_{28} \mathrm{H}_{42} \mathrm{NO}_{6}$ ); see Table 2 for ${ }^{1} \mathrm{H}-,{ }^{13} \mathrm{C}-\mathrm{NMR}\left(600 \mathrm{MHz}, 150 \mathrm{MHz}, \mathrm{CDCl}_{3}\right)$. 


\subsection{Improvement of Antimicrobial Productivity}

In such experiment, the fermentation process was done as described using different culture media (Table 4). The antimicrobial activity of the fermentation clear supernatant was evaluated as described. For quantitative determination of TP in the fermentation supernatant LC-DAD/MS analysis was carried out.

\subsection{LC-DAD/MS Analysis of Trepenomycin and Its Metabolites in Different Media}

\subsubsection{Apparatus and Conditions}

The HPLC system consisted of an Agilent 1200 system, a solvent delivery module, a quaternary pump, an autosampler, a diode-array detector (DAD), and a column compartment (Agilent Technology, Waldbronn, Germany). The column effluent was connected to an Agilent 6320 Ion Trap HPLC-ESI-MS. The column heater was set to $25 \pm 2{ }^{\circ} \mathrm{C}$. The control of the HPLC system and data processing were performed using ChemStation (Rev. B.01.03 SR2(204), Agilent Technology, Waldbronn, Germany) and 6300 Series Trap Control version 6.2 Build No. 62.24 (Bruker Daltonik GmbH, Bremen, Germany). The analytes were separated using an Agilent Zorbax SB-C18 column $\left(80 \mathrm{~A}^{\circ}, 150 \mathrm{~mm}\right.$ length $\times 4.6 \mathrm{~mm}$, i.d., $3.5 \mu$ ) (Agilent Technologies, Palo Alto, CA, USA). The mobile phase was prepared by mixing $760 \mathrm{~mL}$ of $0.1 \%$ ammonia solution $(25 \%, \mathrm{v} / \mathrm{v})$ in water with $240 \mathrm{~mL}$ acetonitrile and was pumped at a flow rate of $0.5 \mathrm{~mL} / \mathrm{min}$. General MS adjustments were set as follows: capillary voltage, $3500 \mathrm{~V}$; nebulizer, $35 \mathrm{psi}$; drying gas, $12 \mathrm{~L} / \mathrm{min}$; desolvation temperature, $350{ }^{\circ} \mathrm{C}$; ion charge control (ICC) smart target, 150,000; and max accumulation time, $150 \mathrm{~ms}$. The UV detector was set at $210 \mathrm{~nm}$. Two MS modes were used, including the negative Auto-MSn scan mode and the single ion monitoring (SIM) mode. The Auto-MSn mode was used to help with the identification of the chemical structures of metabolites, whereas the SIM mode was applied for the quantitative analysis of the parent compound and its diasteriomer. All samples were injected applying negative single ion monitoring at $\mathrm{m} / \mathrm{z} 488.1$ for quantitative analysis. Injection volume was $5 \mu \mathrm{L}$.

\subsubsection{Preparation of Streptomyces Extracts from Different Media for LC-DAD/MS Analysis}

Lyophilized Streptomyces supernatant from different media (200 mg, each) were separately extracted with $2 \mathrm{~mL}$ of methanol in an ultrasonic bath for $20 \mathrm{~min}$. The obtained methanol extracts were centrifuged and the supernatants were passed through 0.45 HPLC membrane filters.

\section{Conclusions}

Streptomyces mutabilis isolated from soil samples in Saudi Arabia produced an anti-tuberculous polyketide macrolide identified as treponemycin (TP). TP also showed a broad spectrum of activity against the Gram positive, Gram negative strains, and Candida albicans. Modification in growth media leads to improvement of TP productivity (150\%) of $S$. mutabilis. During quantitative determination of TP titer by LC/MS after optimization, other metabolites were detected and tentatively identified based on their fragmentation pattern in order to shed light on the differences in metabolites profiling, and not only TP concentration, due to these media changes. Unfortunately, the concentration of these metabolites (T1-T4) was in nano-grams, which make their isolation impossible at this concentration level. Future 
work is currently underway to optimize their concentration to facilitate their isolation and assessment of activity.

\section{Supplementary Materials}

Supplementary materials can be accessed at: http://www.mdpi.com/1420-3049/20/02/2576/s1.

\section{Acknowledgments}

This project is funded by King Abdulaziz City of Science and Technology (KACST), Riyadh, Saudi Arabia, under grant No (A-T-32-64). The authors, therefore, acknowledge with thanks KACST technical and financial support. Also, the authors acknowledge Ikhlas Khan (National Center of Natural Products Research, University of Mississippi, Oxford, MS, USA) for performing HR-EIMS and Alaa Khedre (Faculty of Pharmacy, King Abdulaziz Uiversity, Jeddah, Saudi Arabia) for his unlimited help in LCMS analysis.

\section{Author Contributions}

M.A.Y. and A.A.M.J.-F. collected soil samples, isolated Streptomyces strains, identified the selected Streptomyces isolate, and performed anti-microbial activity. H.M.A. and A.M.E.-H. isolated and identified the bioactive compound. All authors read and approved the final manuscript.

\section{Conflicts of Interest}

The authors declare no conflict of interest.

\section{References}

1. Barkei, J.J.; Kevany, B.M.; Felnagle, E.A.; Thomas, M.G. Investigations into viomycin biosynthesis by using heterologous production in Streptomyces lividans. ChemBiochem 2009, 10, 366-376.

2. Al-Hajoj, S.A. Tuberculosis in Saudi Arabia: Can we change the way we deal with the disease? J. Infect. Public Health 2010, 3, 17-24.

3. Marinelli, F. Antibiotics and Streptomyces: the future of antibiotic discovery-many options for drug discovery are still available. Microbiol. Today 2009, 36, 21-23.

4. Blunt, J.W.; Copp, B.R.; Munro, M.H.; Northcote, P.T.; Prinsep, M.R. Marine natural products. Nat. Prod. Rep. 2006, 23, 26-78.

5. Bull, A.T.; Stach, J.E.; Ward, A.C.; Goodfellow, M. Marine actinobacteria: Perspectives, challenges, future directions. Antonie Leeuwenhoek 2005, 87, 65-79.

6. Berdy, J. Bioactive microbial metabolites. J. Antibiot. 2005, 58, 1-26.

7. Kharat, K.R.; Kharat, A.; Hardikar, B. Antimicrobial and cytotoxic activity of Streptomyces sp. from Lonar Lake. Afr. J. Biotechnol. 2009, 8, 6645-6648.

8. Tanaka, Y.; Kasahara, K.; Hirose, Y.; Murakami, K.; Kugimiya, R.; Ochi, K. Activation and products of the cryptic secondary metabolite biosynthetic gene clusters by rifampin resistance (rpoB) mutations in actinomycetes. J. Biotechnol. 2013, 195, 2959-2970. 
9. Elnakady, Y.A.; Rohde, M.; Sasse, F.; Backes, C.; Keller, A.; Lenhof, H.P.; Weissman, K.J.; Müller, R. Evidence for the mode of action of the highly cytotoxic Streptomyces polyketide kendomycin. ChemBiochem 2007, 8, 1261-1272.

10. Kumagai, T.; Koyama, Y.; Oda, K.; Noda, M.; Matoba, Y.; Sugiyama, M. Molecular cloning and heterologous expression of a biosynthetic gene cluster for the antitubercular agent D-cycloserine produced by Streptomyces lavendulae. Antimicrob. Agents Chemother. 2010, 54, 1132-1139.

11. Stanbury, P.; Whitaker, A.; Hall, S. Media for industrial fermentations. Principles of Fermentation Technology, 2nd ed.; Pergamon: Oxford, UK, 1995; pp. 93-121.

12. Casida, L.E.J. Industrial Microbiology, 2nd ed.; New Age International Limited: New Delhi, India, 1996; pp. 117-134.

13. Nagamitsu, T.; Takano, D.; Marumoto, K.; Fukuda, T.; Furuya, K.; Otoguro, K.; Takeda, K.; Kuwajima, I.; Harigaya, Y.; Omura, S. Total synthesis of borrelidin. J. Org. Chem. 2007, 72, 2744-2756.

14. Cao, Z.; Khodakaramian, G.; Arakawa, K.; Kinashi, H. Isolation of borrelidin as a phytotoxic compound from a potato pathogenic streptomyces strain. Biosci. Biotechnol. Biochem. 2011, 76, 353-357.

15. Maehr, H.; Evans, R.H. Identity of borrelidin with treponemycin. J. Antibiot. 1987, 40, 1455-1456.

16. Saisivam, S.; Bhikshapathi, D.; Krishnaveni, J.; Kishan, V. Isolation of borrelidin from Streptomyces californicus-An Indian soil isolate. Indian J. Biotechnol. 2008, 7, 349-355.

17. Atta, H.M.; El-Sehrawi, M.; Bahobail, A. Antifungal macro-diode production by Streptomyces albidoflavus-143: fermentation, purification and biological activities. J. Am. Sci. 2011, 7, 13-22.

18. Liu, C.-X.; Zhang, J.; Wang, X.-J.; Qian, P.-T.; Wang, J.-D.; Gao, Y.-M.; Yan, Y.-J.; Zhang, S.-Z.; Xu, P.-F.; Li, W.-B. Antifungal activity of borrelidin produced by a Streptomyces strain isolated from soybean. J. Agric. Food Chem. 2012, 60, 1251-1257.

19. Liu, C.; Wang, X.; Yan, Y.; Wang, J.; Zhang, B.; Zhang, J.; Xiang, W. Streptomyces heilongjiangensis sp. nov., a novel actinomycete that produces borrelidin isolated from the root surface of soybean [Glycine $\max ($ L.) Merr]. Int. J. Syst. Evol. Microbiol. 2013, 63, 1030-1036.

20. Lumb, M.; Macey, P.; Spyvee, J.; Whitmarsh, J.; Wright, R. Isolation of vivomycin and borrelidin, two antibiotics with anti-viral activity, from a species of Streptomyces (C2989). Nature 1965, 206, 263-265.

21. Vong, B.G.; Kim, S.H.; Abraham, S.; Theodorakis, E.A. Stereoselective Total Synthesis of (-)-Borrelidin. Angew. Chem. 2004, 116, 4037-4041.

22. Gantt, J.S.; Bennett, C.A.; Arfin, S.M. Increased levels of threonyl-tRNA synthetase in a borrelidin-resistant Chinese hamster ovary cell line. Proc. Natl. Acad. Sci. USA 1981, 78, 5367-5370.

23. Duffey, M.O.; LeTiran, A.; Morken, J.P. Enantioselective total synthesis of borrelidin. J. Am. Chem. Soc. 2003, 125, 1458-1459.

24. Mukhtar, H.; Ijaz, S.; Ikram-ul-Haq. Production of antitumor antibiotic by Streptomyces capoamus. Pak. J. Bot. 2012, 44, 445-452.

25. Anderson, B.; Herlt, A.; Rickards, R.; Robertson, G. Crystal and molecular structures of two isomorphous solvates of the macrolide antibiotic borrelidin: absolute configuration determination by incorporation of a chiral solvent in the crystal lattice. Aust. J. Chem. 1989, 42, 717-730. 
26. Jaradat, Z.; Dawagreh, A.; Ababneh, Q.; Saadoun, I. Influence of culture conditions on cellulase production by Streptomyces sp.(strain J2). Jordan J. Biol. Sci. 2008, 1, 141-146.

27. Clinical and Laboratory Standards Institute. Performance standards for antimicrobial susceptibility testing; Nineteenth Informational Supplement; Document M100-S19; CLSI: Wayne, PA, USA, 2009.

28. Ahmed, I.; Jabeen, K.; Inayat, R.; Hasan, R. Susceptibility testing of extensively drug-resistant and pre-extensively drug-resistant Mycobacterium tuberculosis against levofloxacin, linezolid, and amoxicillin-clavulanate. Antimicrob. Agents Chemother. 2013, 57, 2522-2525.

29. Palomino, J.-C.; Martin, A.; Camacho, M.; Guerra, H.; Swings, J.; Portaels, F. Resazurin microtiter assay plate: Simple and inexpensive method for detection of drug resistance in Mycobacterium tuberculosis. Antimicrob. Agents Chemother. 2002, 46, 2720-2722.

30. Skehan, P.; Storeng, R.; Scudiero, D.; Monks, A.; McMahon, J.; Vistica, D.; Warren, J.T.; Bokesch, H.; Kenney, S.; Boyd, M.R. New colorimetric cytotoxicity assay for anticancer-drug screening. J. Natl. Cancer Inst. 1990, 82, 1107-1112.

Sample Availability: Not available.

(C) 2015 by the authors; licensee MDPI, Basel, Switzerland. This article is an open access article distributed under the terms and conditions of the Creative Commons Attribution license (http://creativecommons.org/licenses/by/4.0/). 